\title{
多面体低聚八(二苯砜基)硅倍半氧烷合成优化及磺酰化机理研究
}

\author{
李紫千杨荣杰* \\ (北京理工大学材料学院 北京 100081)
}

\begin{abstract}
摘要 以笼型低聚八苯基硅倍半氧烷(OPS)为原料, $\mathrm{AlCl}_{3}$ 为催化剂, 苯磺酰氯为磺酰化试剂, 在二氯甲烷溶液中反应 $72 \mathrm{~h}$, 合成了笼型八(二苯砜基)硅倍半氧烷(ODPSS). 该方法合成过程简单稳定, 产率高. 通过 FTIR、MALDI-TOF MS、 ${ }^{1} \mathrm{H}$ NMR、 ${ }^{13} \mathrm{C}$ NMR、 ${ }^{29} \mathrm{Si}$ NMR 和元素分析对产物进行了表征, 证明 OPS 已完全转化为 ODPSS. 通过改变溶剂、催化 剂、反应时间和各反应物物质的量之比，优化了反应条件，分析了反应机理，建立了该体系下 OPS 的磺酰化反应机理 模型.
\end{abstract}

关键词 八(二苯砜基)硅倍半氧烷; 傅-克磺酰化; 八苯基硅倍半氧烷

\section{Optimal Synthesis and Sulfonylation Mechanism of a Novel Polyhedral Oligomeric Octadiphenylsulfonylsilsesquioxane}

\author{
Li, Ziqian Yang, Rongjie* \\ (School of Materials, Beijing Institute of Technology, Beijing 100081)
}

\begin{abstract}
Octadiphenylsulfonylsilsesquioxane (ODPSS) was synthesized through sulfonylation of octaphenylsilsesquioxane (OPS) with benzenesulfonyl chloride and the $\mathrm{AlCl}_{3}$ catalyst in dichloromethane $\left(\mathrm{CH}_{2} \mathrm{Cl}_{2}\right)$. By changing the solvent, catalyst, reaction time and molar ratio of reactants, the optimal reaction conditions were determined. It was found that OPS could be completely converted to ODPSS with a high yield. The product ODPSS was identified by FTIR, MALDI-TOF MS, ${ }^{1} \mathrm{H}$ NMR, ${ }^{13} \mathrm{C}$ NMR, ${ }^{29} \mathrm{Si}$ NMR and elemental analysis. The reaction mechanism was analyzed and a sulfonylation model of OPS was established.
\end{abstract}

Keywords octadiphenylsulfonylsilsesquioxane; Friedel-Crafts sulfonylation; octaphenylsilsesquioxane

由于 POSS 化合物是在分子水平上将无机-有机组 分结合起来, 既保留了无机材料的耐热性和耐氧化性又 兼具有机材料优异的相容性和反应性, 因此其在耐高 温、阻燃、介电材料等领域都有广泛的应用 ${ }^{[1 \sim 8]}$.

POSS 结构单元中的 R 基团可以是多种反应性基团, 如烯基、胺基、环氧基和烷基卤等, 这样 POSS 就可以 通过参与聚合反应对聚合物改性, 如改性环氧树脂、聚 氨酯、聚碳酸酯、聚酰亚胺和聚甲基丙烯酸酯等 ${ }^{[9 \sim 13]}$. $\mathrm{R}$ 基团也可以是非反应基团, 如甲基和异丁基等, 有文献 报道八甲基 POSS 与聚对苯二甲酸乙二酯共混以提高其 阻燃性 ${ }^{[14]}$. 可见, POSS 可以通过共价键或者物理共混 的方式与聚合物相结合, 制备含有 POSS 组分的杂化聚 合物或纳米复合材料. 带反应性基团的 POSS 可使其与 聚合物之间以化学键结合, 实现分子层次上的均匀分
散，近年来被研究者们广泛关注，而对带有非反应性基 团的 POSS 化合物的研究则相对不足. 这可能是因为带 有非反应性基团的 POSS 化合物与聚合物基体的相容性 较差, 难于均匀混合. 当需要应用含非反应性基团的 POSS 化合物时，提高其与聚合物基体的相容性就显得 十分必要. 另外, 聚合物的熔点通常较高, 为了与其熔 融共混, POSS 的热稳定性也同样重要.

苯基硅倍半氧烷作为一种带有非反应基团的 POSS 化合物因其优异的热稳定性而应用于阻燃和耐高温等 领域，但是其很差的溶解性限制了它的应用. 在之前的 工作中, 作者以笼型八苯基倍半硅氧烷 (octaphenylsilsesquioxane, OPS)为反应底物, 经傅-克磺酰化首次合 成了笼型八(二苯砜基)硅倍半氧烷(octadiphenylsulfonylsilsesquioxane, ODPSS), 并充分表征了 ODPSS 的化学

\footnotetext{
*E-mail: yrj@bit.edu.cn

Received May 13, 2014; revised July 6, 2014; published online July 15, 2014.

Project supported by the National Natural Science Foundation of China (No. 512730223).

国家自然科学基金(No. 512730223)资助项目.
} 
结构 ${ }^{[15]}$. ODPSS 在保持 OPS 高热稳定性的同时, 因其在 POSS 分子结构中引入了更多的有机基团, 提高了其溶 解性, 将更有利于提高苯基硅倍半氧烷与聚合物的相容 性. 本文中，作者首次分析了 OPS 的傅克磺酰化反应机 理, 优化了反应条件, 建立了该体系下 OPS 的磺酰化反 应模型.

\section{1 结果与讨论}

\subsection{OPS 傅-克磺酰化反应体系的设计}

OPS 的磺酰化反应是针对该化合物中芳环进行的 亲电取代反应, 为了建立 OPS 的傅-克磺酰化反应体系, 表 1 列举了近年来文献中芳烃的傅-克磺酰化方法. 从 表 1 中可以看出, 傅-克磺酰化的磺酰化试剂常用苯磺 酰氯或对甲苯磺酰氯; 溶剂呈现多样化, 可以是芳烃, 也可以是二氧六环、二氯乙烷等高沸点溶剂, 甚至可用 催化剂代替; 反应温度则基于溶剂的回流温度, 或者略 高于溶剂的回流温度; 催化剂更是研究的热点, 离子溶 液、金属铟和固体酸等各种高效可回收的催化剂为磺酰 化反应提供了更多选择，催化剂效率的不同也直接影响 反应所需的时间.

基于反应物简单易得的原则, OPS 的傅-克磺酰化 反应体系设计如下: 磺酰化试剂为苯磺酰氯; 采用有机 溶剂的均相反应体系, 因为 OPS 为固体粉末, 在无溶剂 条件下与苯磺酰氯反应为非均相反应, 效率较低. 而当 溶剂为二氯甲烷时, OPS 在其中的溶解度相对较高, 反 应近乎均相反应, 当溶剂为三氯甲烷、四氢呋喃、二氧
六环或二氯乙烷时，由于 OPS 在其中的溶解度相对较 低, 反应为非均相反应, 效率较低, 但沸腾回流温度提 高; OPS 与苯磺酰氯和催化剂物质的量之比为 $1: 10$ : 10, 磺酰化试剂和催化剂略过量以保证反应完全; 反应 温度为溶剂的沸腾回流温度，以探索不同温度下反应的 完成情况; 反应时间为 $24 \mathrm{~h}$ ，相对比文献中的反应时间 延长, 以争取 OPS 被完全磺酰化; 催化剂为傅-克反应 传统的催化剂 $\mathrm{AlCl}_{3}$ 和 $\mathrm{FeCl}_{3}$, 因为二者都是强的 Lewis 酸, 催化效率较高而且简单易得.

表 2 列出了合成 ODPSS 的 6 种磺酰化反应方法, 并 利用红外光谱对产物进行了表征(图 1). 从产物的红外 谱图结果分析, 只有方法 1、2 和 5 的产物出现 ODPSS 中的 $\mathrm{O}=\mathrm{S}=\mathrm{O}$ 基团在 $1335 \sim 1295 \mathrm{~cm}^{-1}$ 区域内的不对称 伸缩振动特征峰，而方法 $3 、 4$ 和 6 均没有在该区域内出 现特征吸收峰，可初步判定只有前三个反应中的 OPS 被磺酰化. 进一步对比图 1 中方法 $1 、 2$ 和 5 的红外谱图 可见, 方法 1 中产物 $\mathrm{O}=\mathrm{S}=\mathrm{O}$ 基团特征吸收峰强度最 强, 方法 5 次之, 方法 2 最弱. 而 $\mathrm{O}=\mathrm{S}=\mathrm{O}$ 基团特征吸 收峰的强弱直接反映了产物磺酰化的程度, 即反映了 OPS 转化为 ODPSS 的转化率. 因此可初步判定方法 1 的产物的磺酰化程度最高, 那么该方法就是可以通过继 续优化反应条件以得到高纯度的 ODPSS 的首选方法.

\section{2 新体系的建立与优化}

从方法 1 6 中产物磺酰化的程度可以判定, 当溶 剂为 $\mathrm{CH}_{2} \mathrm{Cl}_{2}$ 、催化剂为 $\mathrm{AlCl}_{3}$ 时反应的转化率最高, 因 此新的体系基于此反应条件，在 $\mathrm{N}_{2}$ 保护和沸腾回流温

表 1 傅-克磺酰化的方法

Table 1 Methods of Friedel-Crafts sulfonylation

\begin{tabular}{|c|c|c|c|c|c|c|c|}
\hline Entry & Catalyst & Sulfonylating agent & Solvent & Reaction time $/ \mathrm{h}$ & $T /{ }^{\circ} \mathrm{C}$ & Atmosphere & Ref. \\
\hline 11 & $\mathrm{FeCl}_{3}$-based ionic liquid & $\begin{array}{l}\mathrm{PhSO}_{2} \mathrm{Cl} \\
\mathrm{TsCl}\end{array}$ & $\mathrm{FeCl}_{3}$-based ionic liquid & $\leqslant 12$ & $\leqslant 165$ & No message & {$[16]$} \\
\hline 2 & $\mathrm{FeCl}_{3}$-based ionic liquid & $\begin{array}{l}\mathrm{PhSO}_{2} \mathrm{Cl} \\
\mathrm{TsCl}\end{array}$ & $\mathrm{FeCl}_{3}$-based ionic liquid & $\leqslant 1$ & 60 & No message & {$[17]$} \\
\hline 3 & $\begin{array}{l}\text { 1-Butyl-3-methylimidazolium } \\
\text { chloroaluminate ionic liquid }\end{array}$ & $\mathrm{TsCl}$ & Ionic liquid & $3,4,5$ & 30,50 & Inert atmosphere & {$[18]$} \\
\hline 4 & $\begin{array}{l}\mathrm{Sn}(\mathrm{OTf})_{2} \\
\mathrm{Cu}(\mathrm{OTf})_{2}\end{array}$ & $\begin{array}{l}\mathrm{PhSO}_{2} \mathrm{Cl} \\
\mathrm{TsCl}\end{array}$ & $\begin{array}{l}\text { Dichloroethane } \\
\text { arene }\end{array}$ & 8,12 & 120 & $\mathrm{~N}_{2}$ & [19] \\
\hline 5 & $\begin{array}{l}\mathrm{Bi}(\mathrm{OTf})_{3} \\
\operatorname{In}(\mathrm{OTf})_{3}\end{array}$ & $\mathrm{MsCl}$ & $\begin{array}{l}\text { Dichloromethane } \\
\text { anisole }\end{array}$ & $4 \sim 6$ & 80 & No message & {$[20]$} \\
\hline 6 & Solid acid & $\begin{array}{l}\mathrm{RSO}_{2} \mathrm{X} \\
\left(\mathrm{R}=\mathrm{CH}_{3}, \mathrm{Ph}, \text { tolyl; }\right. \\
\left.\mathrm{X}=\mathrm{Cl}, \mathrm{OH}, \mathrm{OSO}_{2} \mathrm{R}\right)\end{array}$ & Arene & $3,6,24$ & Reflux & $\mathrm{N}_{2}$ & {$[21]$} \\
\hline 7 & Indium metal & $\begin{array}{l}\mathrm{PhSO}_{2} \mathrm{Cl} \\
\mathrm{TsCl}\end{array}$ & Dioxane & $<3$ & 100 & $\mathrm{~N}_{2}$ & {$[22]$} \\
\hline 8 & $\begin{array}{l}\text { Ps- } \mathrm{AlCl}_{3} \\
\mathrm{SiO}_{2}-\mathrm{AlCl}_{3}\end{array}$ & $\begin{array}{l}\mathrm{PhSO}_{2} \mathrm{Cl} \\
\mathrm{TsCl}\end{array}$ & Arene & $\leqslant 7$ & 85 & No message & {$[23]$} \\
\hline
\end{tabular}


表 26 种 ODPSS 合成方法对比

Table 2 Comparison among synthesis methods of ODPSS

\begin{tabular}{cllc}
\hline Method & Catalyst & \multicolumn{1}{c}{ Solvent } & $\begin{array}{c}\mathrm{O}=\mathrm{S}=\mathrm{O} \text { asymmetric } \\
\text { stretching vibration: } \\
1335 \sim 1295 \mathrm{~cm}^{-1}\end{array}$ \\
\hline 1 & $\mathrm{AlCl}_{3}$ & $\mathrm{CH}_{2} \mathrm{Cl}_{2}$ & Strong \\
2 & $\mathrm{AlCl}_{3}$ & $\mathrm{CHCl}_{3}$ & Very weak \\
3 & $\mathrm{AlCl}_{3}$ & $\mathrm{THF}$ & No peak \\
4 & $\mathrm{AlCl}_{3}$ & $1,4-$ Dioxane & No peak \\
5 & $\mathrm{AlCl}_{3}$ & $1,2-$ Dichloroethane & Weak \\
6 & $\mathrm{FeCl}_{3}$ & $\mathrm{CH}_{2} \mathrm{Cl}_{2}$ & No peak \\
\hline
\end{tabular}

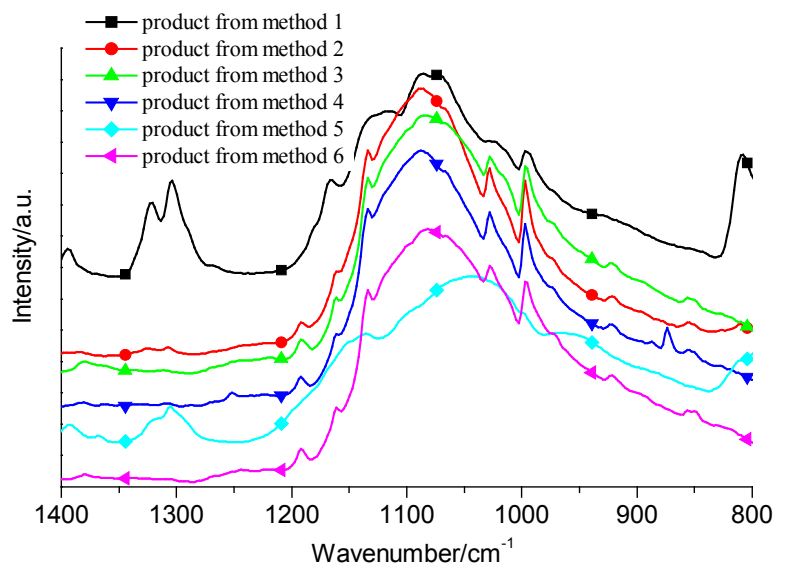

图 1 方法 1 6 产物的红外谱图对比

Figure 1 FTIR spectra of the products from the methods $1 \sim 6$

度下, 通过改变反应时间、OPS 与苯磺酰氯和 $\mathrm{AlCl}_{3}$ 的 物质的量之比来优化反应条件(表 3). 从表 3 中可以看 出, 5 种 ODPSS 合成方法的产率和产物的 $\mathrm{O}=\mathrm{S}=\mathrm{O}$ 基 团特征峰的强度明显受到反应物的物质的量之比和反 应时间的影响. 但反应时间为 $72 \mathrm{~h}$ 时, 产率受到反应物 之间的物质的量之比的影响较小, 产率均较高, 因此推 断反应 $72 \mathrm{~h}$ 后 OPS 的磺酰化进行得相对彻底. 对比方 法 7 11 的 5 种合成方法, 方法 7 的原料投入最少, 在 反应时间为 $72 \mathrm{~h}$ 时, 产率可达到 $86.9 \%$, 因此作为 ODPSS 优选的合成反应体系.

\subsection{ODPSS 的表征}

ODPSS 新合成体系(方法 7, 时间 $72 \mathrm{~h}$ )的确立, 已 成功地多次合成了高纯度的 ODPSS. 图 2 为方法 1 的产 物、方法 $7(72 \mathrm{~h})$ 的产物 ODPSS 与 OPS 的红外谱图对 比图. 从图 2 的红外谱图可以观察到, ODPSS 与 OPS 相 比在 $1322,1304,1136,1070$ 和 $569 \mathrm{~cm}^{-1}$ 处出现了 $\mathrm{O}=$ $\mathrm{S}=\mathrm{O}$ 的特征吸收峰, 在 $810 \mathrm{~cm}^{-1}$ 出现了苯环间位定位 特征吸收峰; 而与方法 1 的产物相比, 方法 7 (72 h) 的产 物 ODPSS 在 1136 和 $1070 \mathrm{~cm}^{-1}$ 处的特征吸收峰的峰型 更加清晰, 没有被 OPS 在 $1088 \mathrm{~cm}^{-1}$ 处的宽频峰所遮蔽, 相对强度更高. 为进一步对比方法 1 的产物和方法 7 (72 h)的产物 ODPSS, 图 3 对比了两者的 MALDI-TOF MS
表 35 种 ODPSS 合成方法的对比与优选

Table 3 Optimization on synthesis methods of ODPSS

\begin{tabular}{|c|c|c|c|c|}
\hline Method & $\begin{array}{c}\text { Molar ratio of OPS : } \\
\text { Benzenesulfonyl } \\
\text { chloride }: \mathrm{AlCl}_{3}\end{array}$ & $\begin{array}{c}\text { Reaction } \\
\text { time } / \mathrm{h}\end{array}$ & Yield/\% & $\begin{array}{c}\mathrm{O}=\mathrm{S}=\mathrm{O} \\
\text { characteristic } \\
\text { absorption } \\
\text { peak }\end{array}$ \\
\hline 7 & $1: 10: 10$ & $\begin{array}{l}48 \\
72\end{array}$ & $\overline{86.9}$ & $\begin{array}{l}\text { Weak } \\
\text { Strong }\end{array}$ \\
\hline 8 & $1: 20: 10$ & $\begin{array}{l}24 \\
48 \\
72\end{array}$ & $\frac{-}{-}$ & $\begin{array}{l}\text { Weak } \\
\text { Weak } \\
\text { Strong }\end{array}$ \\
\hline 9 & $1: 30: 10$ & $\begin{array}{l}24 \\
48 \\
72\end{array}$ & - & $\begin{array}{l}\text { Weak } \\
\text { Weak } \\
\text { Weak }\end{array}$ \\
\hline 10 & $1: 20: 20$ & $\begin{array}{l}24 \\
48 \\
72 \\
96\end{array}$ & $\begin{array}{c}- \\
79.4 \\
85.6 \\
87.1\end{array}$ & $\begin{array}{l}\text { Weak } \\
\text { Strong } \\
\text { Strong } \\
\text { Strong }\end{array}$ \\
\hline 11 & $1: 30: 30$ & $\begin{array}{l}24 \\
48 \\
72 \\
96 \\
\end{array}$ & $\begin{array}{l}- \\
80.4 \\
86.1 \\
85.6\end{array}$ & $\begin{array}{l}\text { Weak } \\
\text { Strong } \\
\text { Strong } \\
\text { Strong }\end{array}$ \\
\hline
\end{tabular}

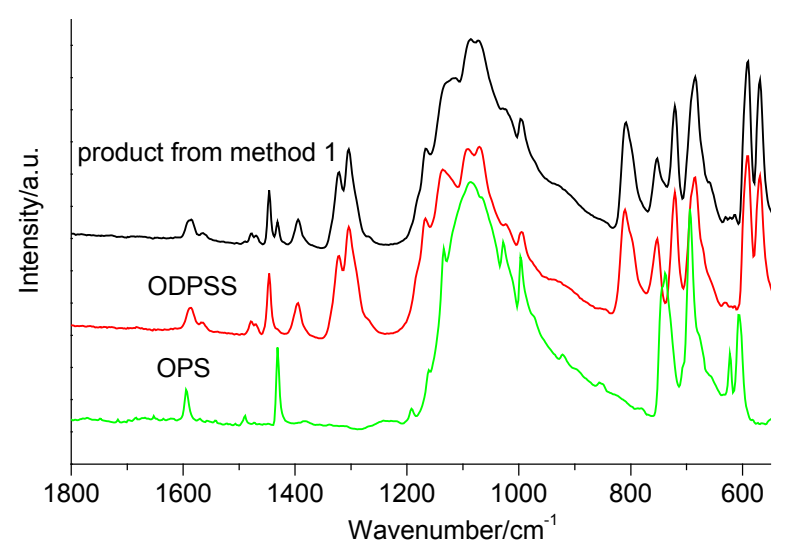

图 2 方法 1 的产物、ODPSS 与 OPS 红外谱图对比

Figure 2 FTIR spectra of the products from the method 1, ODPSS and OPS

图. 图 3 中方法 1 的产物在 1896.2、2036.2 和 2176.3 有 3 个分子离子峰, 分别对应 $6 、 7 、 8$ 三个取代度的笼型 八苯基硅倍半氧烷的磺酰化产物 $\left(+\mathrm{Na}^{+}\right)$, 而方法 7 (72 h)的产物只在 2176.4 处存在一个分子离子峰, 对应 8 取 代的二苯砜基硅倍半氧烷 ODPSS $\left(+\mathrm{Na}^{+}\right)$. 图 4 进一步 对比了方法 1 产物与方法 $7(72 \mathrm{~h})$ 产物 ODPSS 的 ${ }^{1} \mathrm{H}$ NMR 谱图. 在苯环上氢的特征峰共振区, 两者都有 7 组 特征峰: 4 组对应于间位二取代苯环, 3 组对应于单取代 苯环. 其中, ODPSS 的共振峰的积分面积之比为 $1: 1$ : $1: 2: 1: 1: 2$, 符合 8 取代的二苯砜基硅倍半氧烷的 共振峰特征; 而方法 1 产物的共振峰的积分面积之比并 不符合该特征. 另外, 方法 1 产物共振峰的裂分峰数目 
同样与 ODPSS 不同, 不符合 8 取代的二苯砜基硅倍半 氧烷的特征. 结合 MALDI-TOF MS 分析, 方法 1 的产物 是不同取代度的硅倍半氧烷的混合物, ${ }^{1} \mathrm{H}$ NMR 谱图是 各取代度产物谱图叠加的结果. 图 5 为 ODPSS 的 ${ }^{13} \mathrm{C}$ NMR 谱图, 10 个吸收峰对应于 ODPSS 苯环上 10 个处 于不同环境的 $\mathrm{C}$ 原子. 图 6 为 ODPSS 的 ${ }^{29} \mathrm{Si} \mathrm{NMR}$ 谱图,
在 $\delta-79.34$ 处的一个独立的吸收峰代表着硅倍半氧烷 中所有的 $\mathrm{Si}$ 原子都处在相同的环境下，符合 8 取代的二 苯砜基硅倍半氧烷的特征. ODPSS 的 C、H、S 元素分析 表征结果同样与理论值接近. 因此, FTIR, MALDI-TOF $\mathrm{MS},{ }^{1} \mathrm{H} \mathrm{NMR},{ }^{13} \mathrm{C} \mathrm{NMR},{ }^{29} \mathrm{Si} \mathrm{NMR}$ 谱图和元素分析的结 果共同证明了方法 7 (72 h) 对 ODPSS 的成功合成.

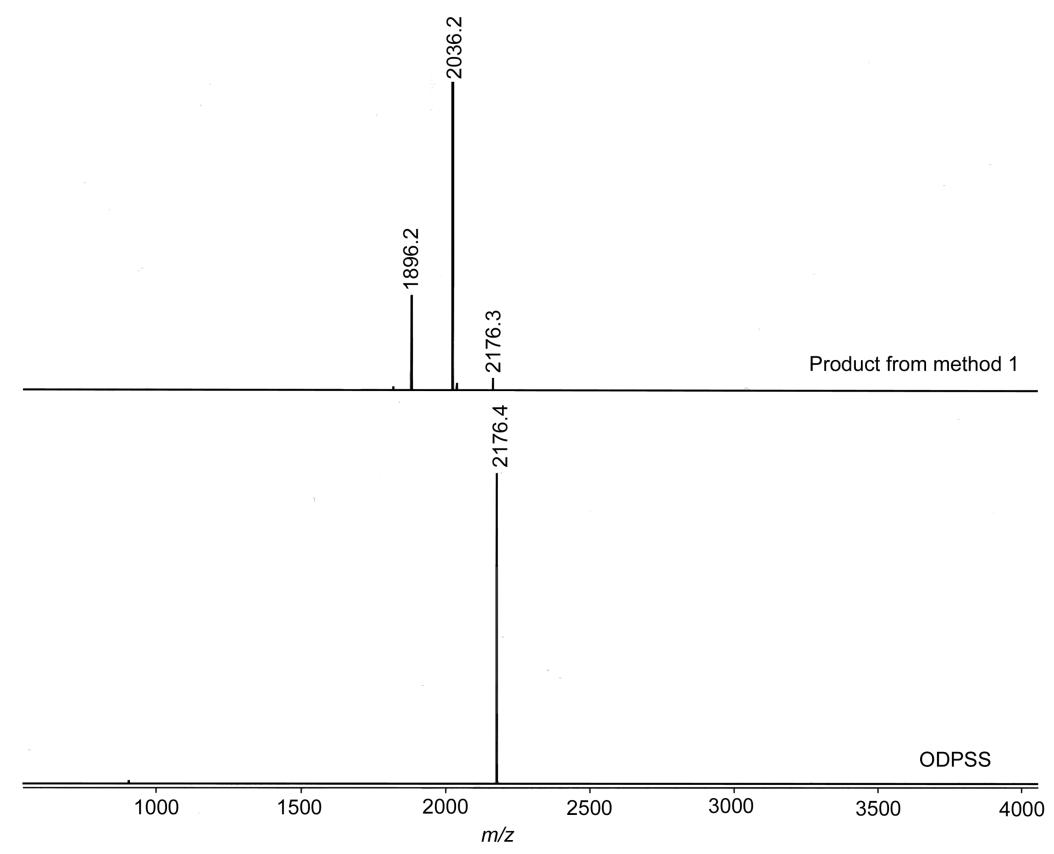

图 3 方法 1 的产物与 ODPSS 的 MALDI-TOF MS 图

Figure 3 MALDI-TOF mass spectra of the products from the method 1 and ODPSS

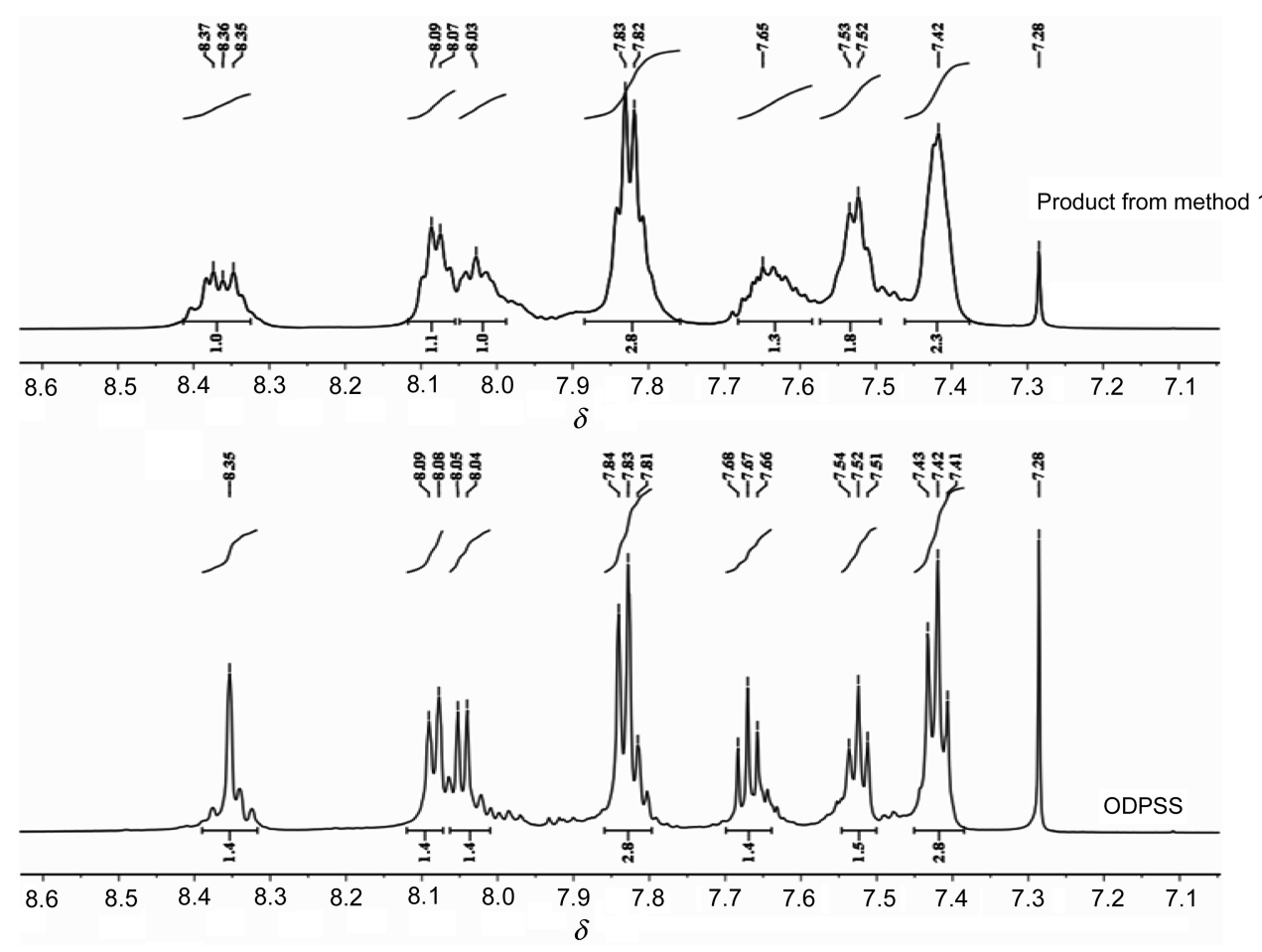

图 4 方法 1 的产物与 ODPSS 的 ${ }^{1} \mathrm{H} \mathrm{NMR}$ 谱图

Figure $4{ }^{1} \mathrm{H}$ NMR spectra of products from the method 1 and ODPSS in $\mathrm{CDCl}_{3}$ 


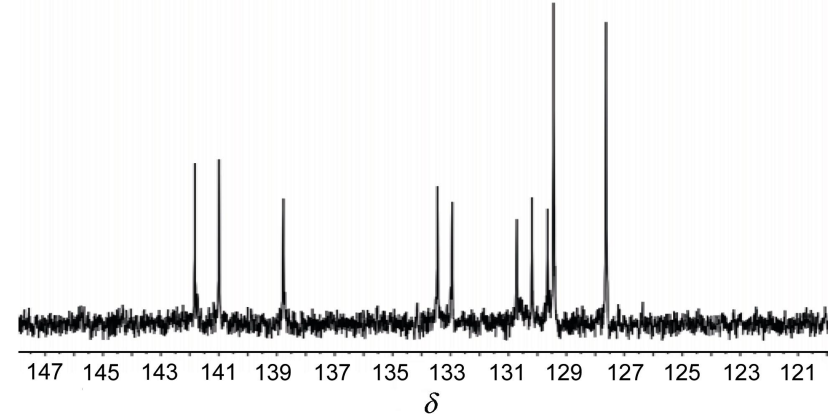

图 5 ODPSS 的 ${ }^{13} \mathrm{C} \mathrm{NMR}$ 谱图

Figure $5{ }^{13} \mathrm{C}$ NMR spectrum of ODPSS in $\mathrm{CDCl}_{3}$

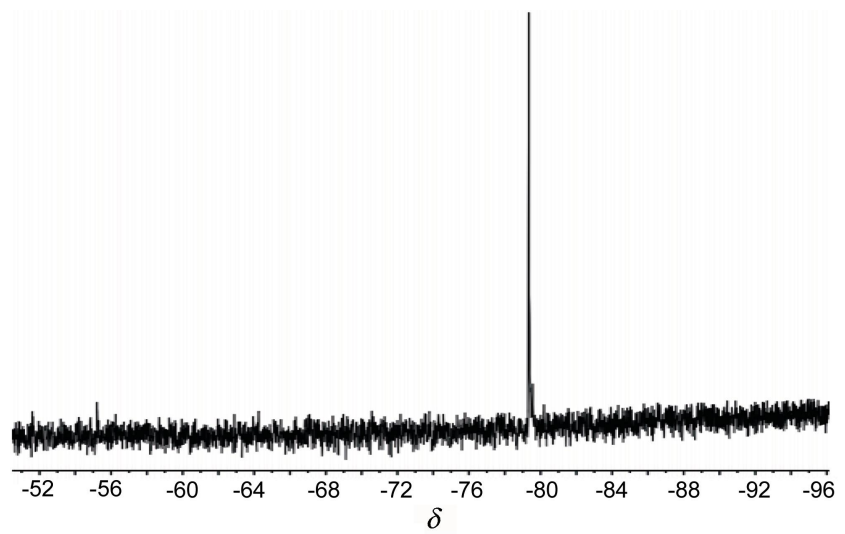

图 6 ODPSS 的 ${ }^{29} \mathrm{Si} \mathrm{NMR}$ 谱图

Figure $6{ }^{29} \mathrm{Si}$ NMR spectrum of ODPSS in $\mathrm{CDCl}_{3}$

1.4 溶剂、催化剂、反应时间、保护气和反应物之间 物质的量之比对反应体系的影响

相比其他溶剂, OPS 在 $\mathrm{CH}_{2} \mathrm{Cl}_{2}$ 中的溶解度最高, 反 应最接近于均相反应, 反应效率最高, 因此在方法 $1 \sim 6$ 中, 方法 1 产物的 $\mathrm{O}=\mathrm{S}=\mathrm{O}$ 基团的特征吸收峰最显著. 可见, 在 OPS 的磺酰化反应体系中, 合适的溶剂是磺酰 化反应顺利进行的关键因素. 同时, $\mathrm{CH}_{2} \mathrm{Cl}_{2}$ 的沸点仅为 $40{ }^{\circ} \mathrm{C}$, 反应体系在较低的温度下即沸腾回流, 有利于 反应产物中 $\mathrm{HCl}$ 气体的排出.

傅-克反应中常用的 Lewis 酸催化剂的催化活性顺 序大致如下: $\mathrm{AlCl}_{3}>\mathrm{FeCl}_{3}>\mathrm{SbCl}_{5}>\mathrm{SnCl}_{4}>\mathrm{BF}_{3}>$ $\mathrm{TiCl}_{4}>\mathrm{ZnCl}_{2}$, 其中 $\mathrm{AlCl}_{3}$ 是效力最强、也是最常用的催 化剂. 对比方法 1 和 6 , 在其他反应条件相同的情况下, 用 $\mathrm{AlCl}_{3}$ 作催化剂时得到目标产物, 而用 $\mathrm{FeCl}_{3}$ 时则没 有得到. 可能是因为 OPS 的磺酰化反应对催化剂的催 化能力要求很高, 具有较强催化能力的 $\mathrm{FeCl}_{3}$ 在方法 6 的反应条件下并没有成功催化 OPS 得到其磺酰化产物.

方法 1 和 7 的不同点仅在于方法 7 延长了反应时间， 而对比产物的表征数据可知, 延长反应时间有利于提高 产物中 ODPSS 的含量, 使磺酰化反应进行得更加彻底. 当反应时间为 $72 \mathrm{~h}$ 时, MALDI-TOF MS 的表征结果显 示产物为单一的八个二苯砜基取代的硅倍半氧烷.
为了研究保护气对反应的影响, 针对方法 10 设计 了一个对比实验 12: 其他实验条件相同，仅在反应开始 $1 \mathrm{~h}$ 后, 停止通入 $\mathrm{N}_{2}$ (将进气口用瓶塞塞紧, 出气口与空 气相通，保持体系内气体不经吹扫而自行排出), 对比 表征反应 $72 \mathrm{~h}$ 后产物的红外光谱图(图 7). 从产物的红 外光谱图中可以看出，在 $\mathrm{N}_{2}$ 保护下的产物 $\mathrm{O}=\mathrm{S}=\mathrm{O}$ 基 团的红外特征峰形更尖锐, 峰的强度更高, 对应产物中 的 ODPSS 的含量更高. 可见保护气能吹扫反应生成的 $\mathrm{HCl}$ 气体，有利于反应向高磺酰化程度进行.

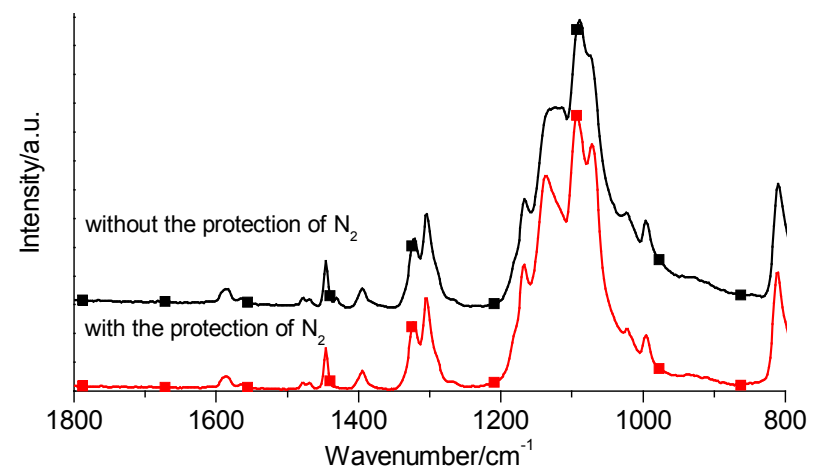

图 $7 \mathrm{~N}_{2}$ 保护与非 $\mathrm{N}_{2}$ 保护下产物的红外谱图对比

Figure 7 FTIR spectra of the products with and without the protection of $\mathrm{N}_{2}$

同时增大磺酰化试剂和催化剂的用量(表 3), 在反 应 $48 \mathrm{~h}$ 即可得到可通过红外表征的 ODPSS, 但当反应 时间延长至 $72 \mathrm{~h}$ 时，与其他方法相比产物 ODPSS 的产 率并没有提高; 单纯提高磺酰化试剂的用量而 $\mathrm{AlCl}_{3}$ 的 用量不变时, 如 OPS、苯磺酰氯与 $\mathrm{AlCl}_{3}$ 的物质的量之 比为 $1: 20 ： 10$ 时, 通过了红外表征; 继续增大磺酰化 试剂用量, 当三者的物质的量之比为 $1: 30: 10$ 时, 则 无法通过红外表征. 可见, 磺酰化试剂与催化剂之间存 在一个的优选比例 $(1: 10: 10)$, 适当的比例有利于反 应生成高磺酰化程度的产物.

\subsection{ODPSS 的磺酰化反应机理分析}

傅一克酰基化反应中，磺酰化反应机理在文献中的 报道较少. 一般认为, 傅-克磺酰化反应中的酰化试剂 在催化剂的作用下，首先生成酰基正离子，然后其与芳 环发生亲电取代 ${ }^{[24,25]}$. 因此, 推测当磺酰化试剂为苯磺 酰氯时苯的傅-克磺酰化反应机理如 Scheme 1 所示. 由 此机理推断，当磺酰化试剂过量时，利于磺酰基正离子 的形成, 进一步有利于苯环上亲电取代的发生. 但这并 不能解释 OPS 的磺酰化反应中当苯磺酰氯相对于 OPS 和 $\mathrm{AlCl}_{3}$ 大过量 $(1: 30: 10)$ 时, 傅-克磺酰化反应程度 反而降低. 据此推断与羰基类似，苯磺酰氯中的砜基同 样能与 $\mathrm{AlCl}_{3}$ 络合 (Scheme 2), 消耗催化剂的量, 从而降 低磺酰化的反应程度. 但是与羰基不同的是，这种络合 
物没有羰基与 $\mathrm{AlCl}_{3}$ 形成的络合物稳定(1 个羰基络合一 个 $\mathrm{AlCl}_{3}$, 而 1 个砜基不一定能络合 2 个 $\mathrm{AlCl}_{3}$ ). 砜基上 的氧原子对称、无极性, 两个氧原子提供孤对电子的能 力较崖基上氧原子的能力弱, 因此砜基与 $\mathrm{AlCl}_{3}$ 的络合 几率低, 这可由方法 7、8、10、11 的高磺酰化反应程度 证实. 只有当砜基大过量时, 才会明显消耗催化剂 $\mathrm{AlCl}_{3}$ 的量, 从而降低磺酰化的反应程度 (方法 9). 方法 12 中由于没有了 $\mathrm{N}_{2}$ 吹扫反应出的 $\mathrm{HCl}$ 气体, 导致体系 中 $\mathrm{HCl}$ 浓度过高影响了 Scheme 1 中(3)的反应速率, 在 $72 \mathrm{~h}$ 内未生成高磺酰化程度的产物.

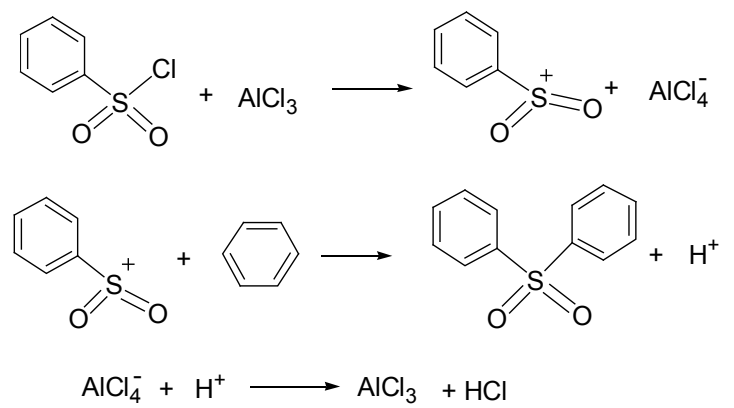

Scheme 1<smiles>CC(Cl)(Cl)COP(=O)(Cl)c1ccccc1</smiles>

Scheme 2

\subsection{OPS 磺酰化反应模型}

基于以上 OPS 的磺酰化反应机理的分析，提出了
以下的 OPS 与苯磺酰氯在 $\mathrm{AlCl}_{3}$ 催化作用下的磺酰化反 应模型(Scheme 3). 首先, 部分苯磺酰氯与 $\mathrm{AlCl}_{3}$ 形成络 合物消耗掉部分催化剂, 余下的 $\mathrm{AlCl}_{3}$ 与苯磺酰氯形成 锍离子作为亲电试剂进攻 OPS 上的苯环. 锍离子从苯 环的 $\pi$ 体系中得到两个 $\pi$ 电子，与苯环上一个碳原子形 成 $\sigma$ 键, 生成 $\sigma$ 络合物. 此时, 这个碳原子由 $\mathrm{sp}^{2}$ 杂化变 成 $\mathrm{sp}^{3}$ 杂化状态, $\sigma$ 络合物很容易从 $\mathrm{sp}^{3}$ 杂化碳原子上失 去一个质子，使该碳原子恢复成 $\mathrm{sp}^{2}$ 杂化状态，再形成 六个 $\pi$ 电子离域的闭合共轭体系, 生成取代苯. 这个失 去的 $\mathrm{H}^{+}$将 $\mathrm{Cl}^{-}-\mathrm{AlCl}_{3}$ 氧化成 $\mathrm{AlCl}_{3}$ 催化该磺酰化反应继 续进行. 这样当苯磺酰氯大过量时, 其与 $\mathrm{AlCl}_{3}$ 形成了 相对多的络合物, 消耗了作为催化剂的 $\mathrm{AlCl}_{3}$ 的量, 这 也导致亲电试剂锍离子的数量减少，从而降低了整个反 应的磺酰化速率.

\section{2 结论}

OPS 与苯磺酰氯在 Lewis 酸的催化作用下经过傅克磺酰化反应制备 ODPSS 的体系中, 通过改变溶剂、 催化剂、反应时间，最终确定的反应体系为：以 OPS 为 原料, 苯磺酰氯为磺酰化试剂, $\mathrm{AlCl}_{3}$ 为催化剂, 三者物 质的量之比为 $1: 10: 10$, 在二氯甲烷溶剂中沸腾回流 反应 $72 \mathrm{~h}$. 此体系原料易得, 合成过程简单且稳定, 产 率高. 通过 FTIR、MALDI-TOF MS、 ${ }^{1} \mathrm{H}$ NMR、 ${ }^{13} \mathrm{C}$ NMR、 ${ }^{29} \mathrm{Si} N M R$ 和元素分析对产物进行了表征, 证明得到了 目标产物 ODPSS. 分析了 OPS 的磺酰化反应机理, 建 立了 OPS 在该体系下的磺酰化反应模型：苯磺酰氯消 耗部分 $\mathrm{AlCl}_{3}$ 与之形成络合物, 余下的 $\mathrm{AlCl}_{3}$ 再与苯磺酰

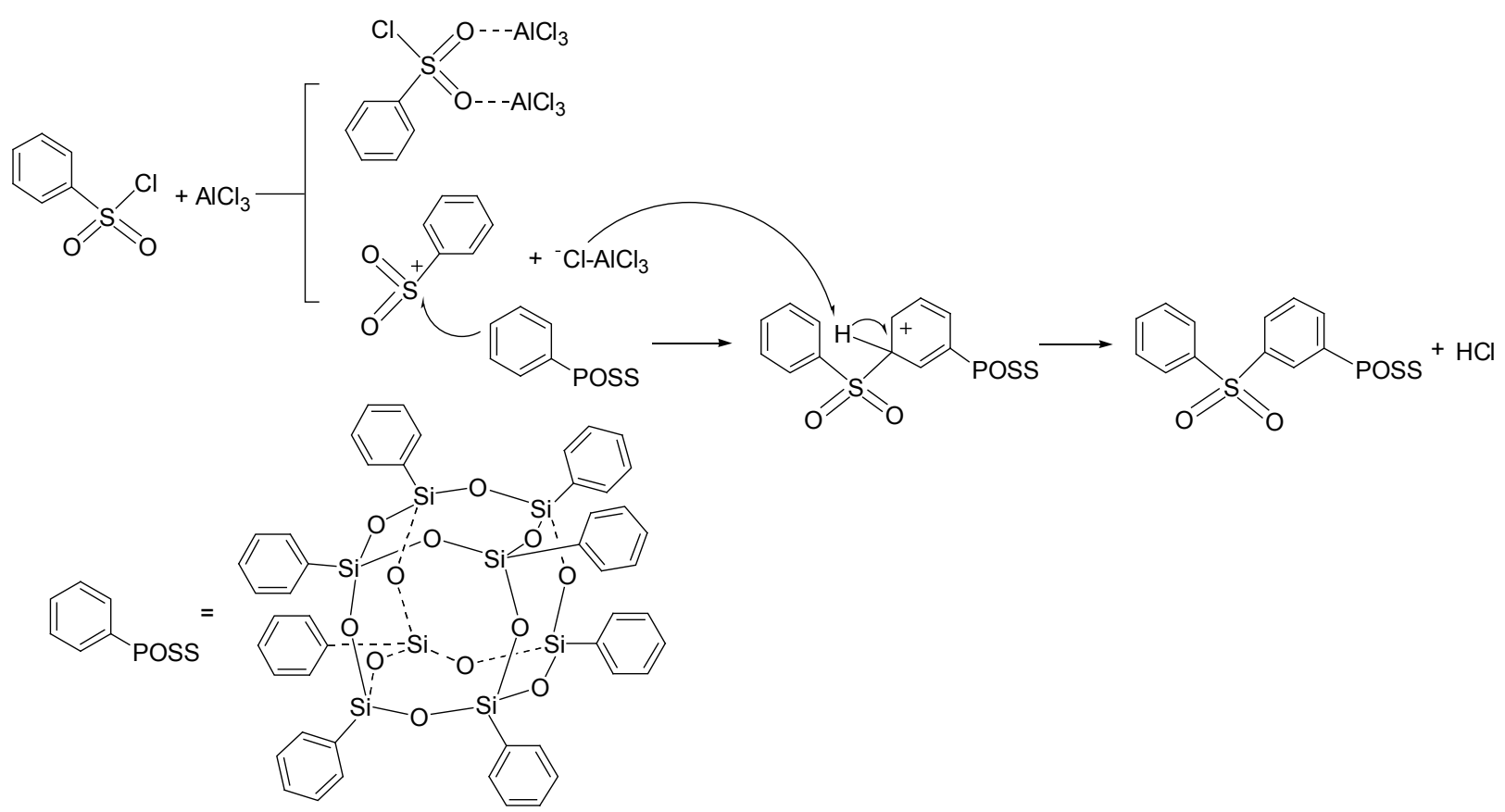

Scheme 3 
氯形成锍离子和 $\mathrm{AlCl}_{4}^{-}$. 该锍离子作为亲电试剂进攻 OPS 上的苯环形成 $\sigma$ 络合物, 随后 $\sigma$ 络合物失去一个 $\mathrm{H}^{+}$将 $\mathrm{AlCl}_{4}^{-}$氧化成 $\mathrm{AlCl}_{3}$ 催化该磺酰化反应继续进行.

\section{3 实验部分}

\section{1 仪器与试剂}

红外分析(FTIR): 美国 Nicolet 6700 FTIR 傅立叶变 换红外仪, 分辨率为 $4 \mathrm{~cm}^{-1}$, 扫描次数为 32 , 光谱采集 速率为 0.6329 , 检测器类型为 $\mathrm{DTGS} / \mathrm{KBr}$; 核磁共振分 析: 德国 Bruker Avance 600 NMR 核磁共振谱仪; 元素 分析: 德国 Vario EL III 元素分析仪; 质谱: Bruker BIFLEX III 型基质辅助激光解析电离飞行时间质谱.

八苯基硅倍半氧烷参照文献[4]方法合成; 苯磺酰 氯：阿拉丁试剂(上海)有限公司; 二氯甲烷、乙醇、正己 烷、三氯化铝均为市售分析纯.

\section{2 实验方法}

ODPSS 的合成过程如 Eq. 1 所示, 称取 $10.34 \mathrm{~g}$ OPS(相当于 $80 \mathrm{mmol}$ 苯基)加入带回流冷凝管、恒压滴 液漏斗和氮气保护的 $250 \mathrm{~mL}$ 的三口烧瓶中, 用 $150 \mathrm{~mL}$ 二氯甲烷溶解, 然后加入无水三氯化铝 $13.34 \mathrm{~g}$ (100 $\mathrm{mmol}$ )加热升温至 $40{ }^{\circ} \mathrm{C}$, 开始缓慢滴加 $17.7 \mathrm{~g}$ 苯磺酰氯 (100 mmol), 约 $30 \mathrm{~min}$ 滴完, 滴加过程中逐渐放出大量 气体, 滴加完毕后, 回流反应 $72 \mathrm{~h}$. 反应完毕后将反应 液倒入盛有 $100 \mathrm{~g}$ 碎冰的烧杯中, 再加入 $500 \mathrm{~mL}$ 正已烷 搅拌, 沉淀. 过滤掉液体, 将沉淀溶于 $100 \mathrm{~mL}$ 二氯甲 烷, 然后倾入 $1 \mathrm{~L}$ 冷乙醇中, 析出白色沉淀物, 抽滤, 用 $3 \mathrm{~L}$ 去离子水洗沉淀, 真空烘箱 $80{ }^{\circ} \mathrm{C}$ 干燥 $24 \mathrm{~h}$. 得到产 物 $18.718 \mathrm{~g}$, 产率 $86.9 \%$. ${ }^{1} \mathrm{H} \mathrm{NMR}\left(600 \mathrm{MHz}, \mathrm{CDCl}_{3}\right) \delta$ : $7.41 \sim 7.43$ (t, $16 \mathrm{H}, \mathrm{H}$ meta to $\left.\mathrm{SO}_{2} \mathrm{Ph}\right), 7.51 \sim 7.54(\mathrm{t}, 8 \mathrm{H}$, meta to both POSS and $\left.\mathrm{SO}_{2} \mathrm{Ph}\right), 7.66 \sim 7.68(\mathrm{t}, 8 \mathrm{H}$, para to $\mathrm{SO}_{2} \mathrm{Ph}$ ), $7.83 \sim 7.84$ (d, $16 \mathrm{H}$, ortho to $\mathrm{SO}_{2} \mathrm{Ph}$ ), 8.04 8.05 (d, $8 \mathrm{H}$, ortho to $\mathrm{SO}_{2} \mathrm{Ph}$ and para to POSS), 8.08 8.09 (d, $8 \mathrm{H}$, ortho to POSS and para to $\left.\mathrm{SO}_{2} \mathrm{Ph}\right), 8.35$ (s, $8 \mathrm{H}$, ortho to both POSS and $\left.\mathrm{SO}_{2} \mathrm{Ph}\right) ;{ }^{13} \mathrm{C} \mathrm{NMR}\left(151 \mathrm{MHz}, \mathrm{CDCl}_{3}\right) \delta$ : $141.83\left(\mathrm{CSO}_{2} \mathrm{Ph}\right), 140.99\left(\mathrm{CSO}_{2} \mathrm{Ph}\right), 138.77,133.44$,

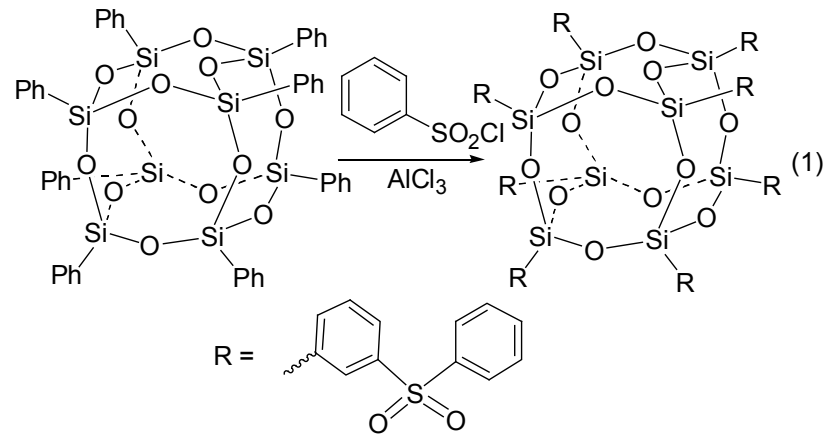

$132.95,130.71,130.19,129.65,129.44,127.63 ;{ }^{29} \mathrm{Si}$ NMR $\left(119 \mathrm{MHz}, \mathrm{CDCl}_{3}\right) \delta$ : $-79.34\left(\mathrm{SiPhSO}_{2} \mathrm{Ph}\right)$; FTIR $v$ : 1322, 1304, 1136, 1070, $569(\mathrm{O}=\mathrm{S}=\mathrm{O}), 1088$ (broad, $\mathrm{Si}-\mathrm{O}-\mathrm{Si}), 810(\mathrm{~m}-\mathrm{CH}) \mathrm{cm}^{-1}$; MALDI-TOF MS $m / z(\%)$ : $2176.4\left(\mathrm{M}+\mathrm{Na}^{+}, 100\right)$. Anal. calcd for ODPSS: C 53.51, H 3.37, S 11.90; found C 52.55, H 3.82, S 11.58.

\section{References}

[1] Zhang, L.-L.; Liu, A.-H.; Zeng, X.-R. Chin. J. Org. Chem. 2007, 27,424 (in Chinese).

(张利利, 刘安华, 曾幸荣, 有机化学, 2007, 27, 424.)

[2] Cordes, D. B.; Lickiss, P. D.; Rataboul, F. Chem. Rev. 2010, 110, 2081.

[3] Wang, X.-L.; Liang, G.-Z.; Zhang, Z.-P. Mater. Rev. 2007, 21, 34 (in Chinese).

(王晓蕾, 梁国正, 张增平，材料导报，2007, 21, 34.)

[4] Li, L.; Li, X.; Yang, R. J. Appl. Polym. Sci., 2012, 124, 3807.

[5] Zhang, W.; Li, X.; Yang, R. Polym. Degrad. Stab. 2011, 96, 2167.

[6] Cai, H.; Zhang, X.; Xu, K.; Liu, H.; Su, J.; Liu, X.; Fu, Z.; Guo, Y.; Chen, M. Polym. Adv. Technol. 2012, 23, 765.

[7] Leu, C.-M.; Chang, Y.-T.; Wei, K.-H. Macromolecules 2003, 36, 9122.

[8] Hou, Z.-Y.; Hu, M.-B.; Wang, W. Acta Chim. Sinica 2014, 72, 61 (in Chinese).

(侯占要, 胡敏标, 王维, 化学学报, 2014, 72,61.)

[9] Zhang, W.; Li, X.; Yang, R. Polym. Degrad. Stab. 2011, 96, 2167.

[10] Jost, C.; Kuehnle, A.; Abbenhuis, H. C. L. WO 03042223, 2003 [Chem. Abstr. 2003, 138, 385570].

[11] Nagendiran, S.; Alagar, M.; Hamerton, I. Acta Mater. 2010, 58, 3345.

[12] Iyer, P.; Coleman, M. R. J. Appl. Polym. Sci. 2008, 108, 2691.

[13] An, Y.-C.; Jeon, J.-H.; Lee, S. W.; Min, B.-G.; Lim, J.-H.; Kim, K.-M. Polym. J. (Tokyo, Jpn.) 2009, 41, 303.

[14] Vannier, A.; Duquesne, S.; Bourbigot, S.; Castrovinci, A.; Camino, G.; Delobel, R. Polym. Degrad. Stab. 2008, 93, 818.

[15] Li Z.; Yang. R. J. Appl. Polym. Sci. 2014, 131, 10375.

[16] Alexander, M. V.; Khandekar, A. C.; Samant, S. D. J. Mol. Catal. A: Chem. 2004, 223, 75.

[17] Bahrami, K.; Khodei, M. M.; Shahbazi, F. Tetrahedron Lett. 2008, 49, 3931.

[18] Nara, S. J.; Harjani, J. R.; Salunkhe, M. M. J. Org. Chem. 2001, 66, 8616.

[19] Singh, R. P.; Kamble, R. M.; Chandra, K. L.; Saravanan, P.; Singh, V. K. Tetrahedron 2001, 57, 241 .

[20] Wallace, M. A.; Raab, C.; Dean, D.; Melillo, D. J. Labelled Compd. Radiopharm. 2007, 50, 347.

[21] Choudary, B. M.; Chowdari, N. S.; Kantam, M. L. J. Chem. Soc., Perkin Trans. 1 2000, 2689.

[22] Jang, D. O.; Moon, K. S.; Cho, D. H.; Kim, J.-G. Tetrahedron Lett. 2006, 47, 6063.

[23] Borujeni, K. P.; Tamami, B. Catal. Commun. 2007, 8, 1191.

[24] Huang, Z.-L.; Jin, L.-Q.; Lei, A.-W. Chin. J. Org. Chem. 2011, 31, 775 (in Chinese). (黄志良, 靳立群, 雷爱文, 有机化学, 2011, 31, 775.)

[25] Smith, M. B.; March, J. March's Advanced Organic Chemistry: Reactions, Mechanisms, and Structure, 6th ed., Wiley-Interscience, New York, 2007 